\title{
The Novel Use of Cerebrolysin and Citicoline in the Treatment of Kernicterus
}

\author{
Aamir Jalal Al Mosawi* \\ Advisor in Pediatrics and Pediatric Psychiatry, Children Teaching Hospital of Baghdad Medical City, Iraq \\ *Corresponding author: Aamir Jalal Al Mosawi, Advisor in Pediatrics and Pediatric Psychiatry, Children Teaching Hospital of \\ Baghdad Medical City, Iraq
}

\begin{abstract}
Background: There is no effective therapy for many neurological disorders associated with significant neurological damage such as kernicterus. Patients with Kernicterus (Chronic bilirubin encephalopathy) which is a neurological dysfunction resulting from exposure of the brain to severe hyperbilirubinemia mostly during the neonatal period continue to experience significant disability. There is no known therapeutic intervention that can obviously improve this condition. The aim of this paper is to describe a novel therapeutic approach which in two months produced marked improvement of the neurological dysfunction caused by kernicterus.

Patients and Methods: A girl with kernicterus was not speaking and not saying any word. She was lacking the balance (coordination) without obvious muscle weakness. She was unable to maintain the sitting posture on a chair for few minutes. She was unable to maintain straight standing posture when supported on chair at all. She had difficulty in holding things. The girl was treated with a novel therapeutic approach including two courses of intramuscular cerebrolysin and intramuscular citicoline.

Results: After treatment the first month of treatment, speech development was initiated, and she was saying few words. She was able to sit normally on the chair and maintaining the sitting posture indefinitely. She was able to maintain more straight stable standing posture without holding a chair and with the ability to hold things at the same time indicting improved coordination. She also developed improved ability to hold small things like a pen. After the second month of treatment, the girl was able to stand alone and was making few steps slowly holding furniture. After treatment the second course of treatment, the girl was able to stand alone and walk rapidly holding furniture. Treatment was not associated with any side effects.
\end{abstract}

Conclusion: The novel use of intramuscular cerebrolysin and citicoline in a patient with kernicterus was safe and effective.

Keywords: Cerebrolysin; Citicoline Treatment; Kernicterus

\section{Introduction}

Kernicterus is a neurological dysfunction resulting from exposure of the brain to severe hyperbilirubinemia mostly during the neonatal period. In kernicterus, hyperbilirubinemia results in deposition of bilirubin in the grey matter of the brain causing neurotoxicity associated with mass-destruction of neurons by apoptosis and necrosis resulting in irreversible brain damage, and chronic neurological disorder. Clinical manifestations of kernicterus may include severe motor disability with inability to walk. There is no effective therapy kernicterus [1,2]. The aim of this paper is to describe a novel therapeutic approach which in two moths produced marked improvement of the neurological dysfunction caused by kernicterus.

\section{Patients and Method}

A girl was first seen at the age of about five years during November,2018 because of delayed development with lack of body control, abnormal movements, and no speech. Her birth weight was about three Kilogram, and she developed severe neonatal hyperbilirubinemia of $26 \mathrm{mg} / \mathrm{dL}$, and was treated by phototherapy and exchange transfusion. The family consulted many physicians in Iraq, and when no obvious improvement could be achieved nor any hope of improvement was given, the family took their daughter to India. During March,2017, the girl was seen by Dr Rakesh Kumar Jain who was a senior consultant at the neurology center of Fortis Memorial Research Institute in Gurgaon India. Dr Jain 
performed a 21-channel electroencephalography on the girl which showed intermittent short and sharp waves from bilateral focal regions with no ictal events. The electroencephalography report stated that drug induced EEG showed normal sleep pattern with intermittent bilateral frontal sharp waves. Dr Jain also performed a study of the short latency auditory evoked potential on the girl. The report of this study stated that the stem auditory evoked response showed normal latencies of waves with hearing threshold of $20 \mathrm{~dB}$ bilaterally. Dr Jain sent the girl to the Modern Diagnostic Research Center for brain MRI which was performed at the $22^{\text {nd }}$ of March, 2017, by Dr Sneha Thakur, a consultant radiologist. The brain MRI detected no significant abnormality. Dr Rupal Gupta, a consultant ophthalmologist at the Fortis Memorial Research Institute examined the girl on the $30^{\text {th }}$ of March, 2017, and prescribed eye glasses for the girl. After visiting many physicians in Iraq and India, the only beneficial therapeutic intervention for the girl was the correction of her refractive error with eyeglasses.

When the girl was seen at the pediatric neuropsychiatry clinic at the Children Teaching Hospital of Baghdad Medical City during November,2018, she was not speaking and was not saying any word. The girl was lacking the balance (co-ordination) without obvious muscle weakness. She was unable to maintain the sitting posture on a chair for few minutes and was also unable to maintain straight standing posture when supported on chair at all (Figure 1). She also had difficulty in holding things.

The girl was treated with a novel therapeutic approach consisting of two courses.

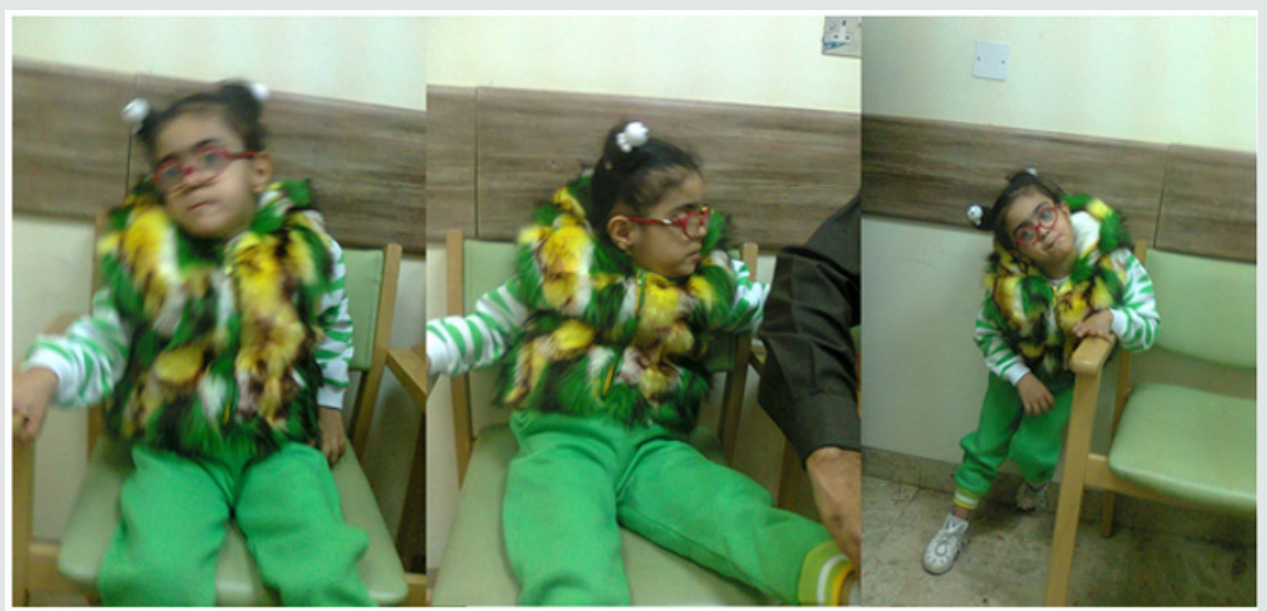

Figure 1: The girl with kernicterus before treatment.

\section{The First Course Included}

Cerebrolysin $5 \mathrm{ml}$ given by intra-muscular injections every third day on the morning, and she received 20 doses over two months. Citicoline $500 \mathrm{mg}$ given by intra-muscular injections every third day on the morning, and she received 20 doses over two months. Cerebrolysin and citicoline were given on two different days.

\section{The Second Course Included}

Cerebrolysin $5 \mathrm{ml}$ given by intra-muscular injections every fifth day on the morning, and she received 10 doses over fifty days. Citicoline $500 \mathrm{mg}$ given by intra-muscular injections every fifth day on the morning, and she received 10 doses over fifty days. Cerebrolysin and citicoline were given on two different days.

\section{Results and Discussion}

After treatment the first month of treatment, speech development was initiated and she was saying few words. She was able to sit normally on the chair and maintaining the sitting posture indefinitely (Figure 1). She was able to maintain more straight stable standing posture without holding a chair and with the ability to hold things at the same time indicting improved coordination (Figure 2A). She also developed improved ability to hold small things like a pen (Figure 2B). After the second month of treatment, the girl was able to stand alone and was making few steps slowly holding furniture. After treatment the second course of treatment, the girl was able to stand alone and walk rapidly holding furniture (Figure 3). Treatment was not associated with any side effects. In this paper, we have reported that the novel use of intramuscular cerebrolysin and citicoline in a patient with kernicterus was safe and effective. The use of cerebrolysin and citicoline was beneficial in the treatment of various childhood neuro-psychiatric disorders including developmental and pervasive developmental disorders, brain atrophy, kernicterus, and cerebral palsy [3-5]. Cerebrolysin is a peptidergic medicine which contains mainly biologically active neuropeptides including brain-derived neurotrophic factor, glial cell line-derived neurotrophic factor, nerve growth factor, and ciliary neurotrophic factor. It has a nerve growth factor like activity on neurons, and growth promoting efficacy in different neuronal populations from peripheral and central nervous system. Cerebrolysin has a direct neurotrophic effect, and obvious neuroprotective properties against many types of lesion in vitro and in vivo. The therapeutic effects of cerebrolysin have been considered to be similar to the pharmacological activities of naturally occurring nerve growth factors. The safety, tolerability, and efficacy of neuroreparative cerebrolysin therapy have been established in clinical trials included adults with stroke and Alzheimer s disease. 

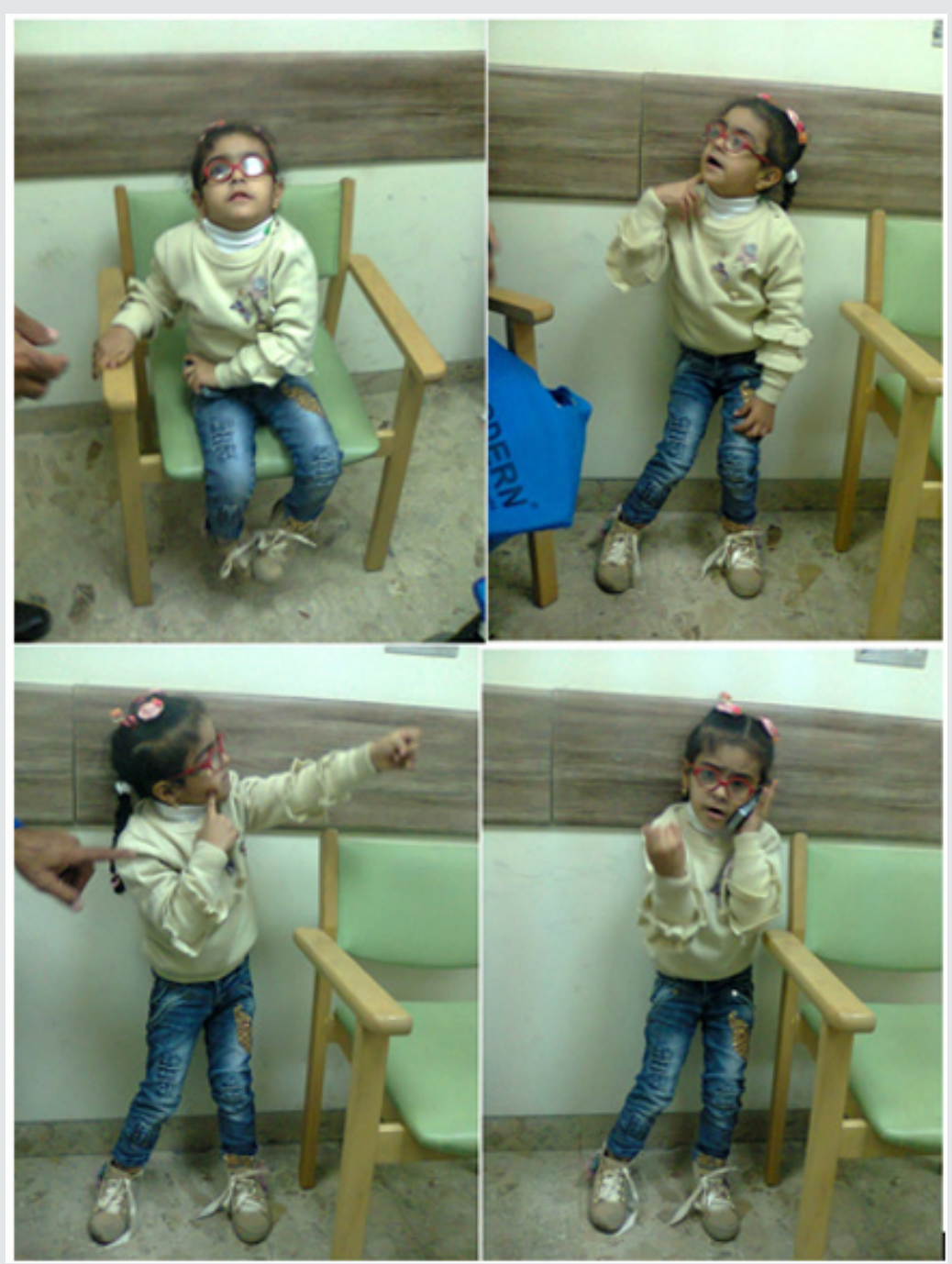

Figure 2A: The girl with kernicterus before treatment.

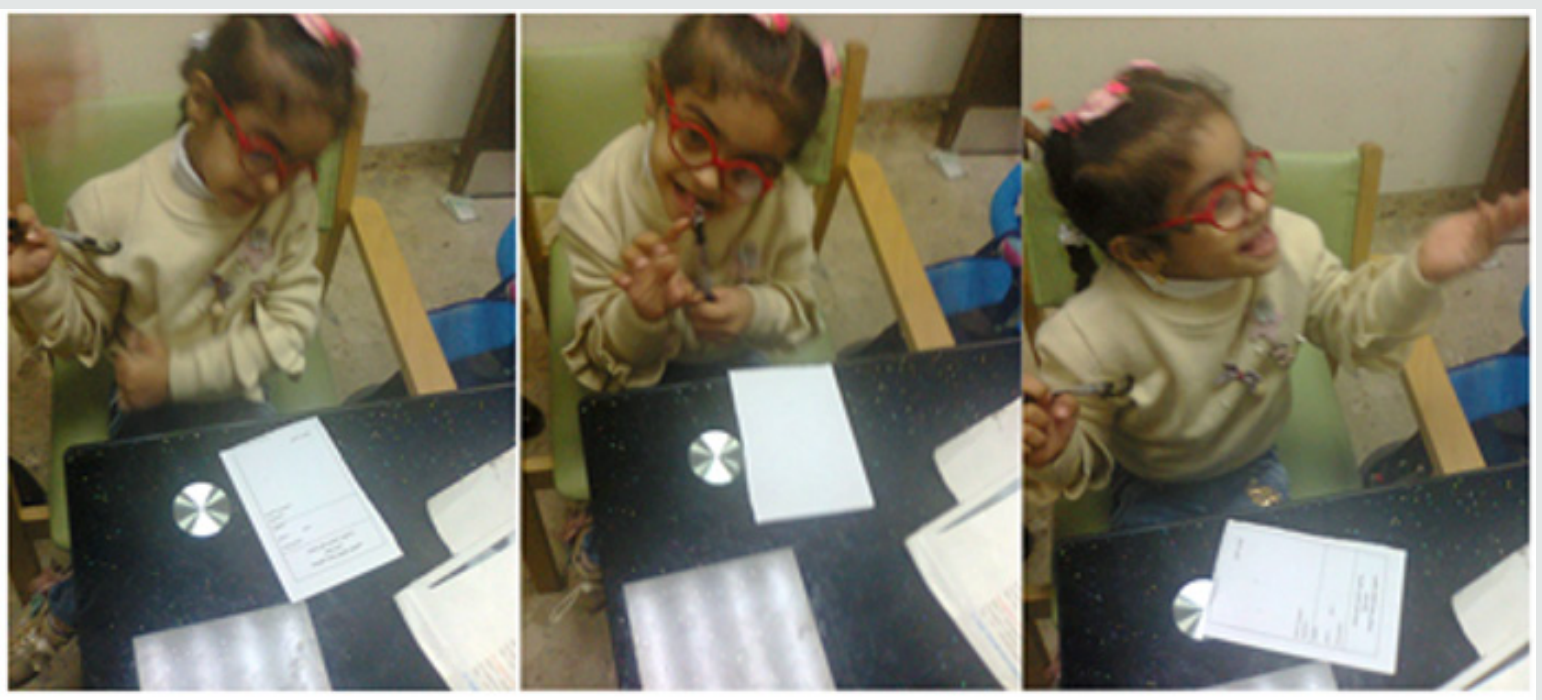

Figure 2B: The girl with kernicterus after two months treatment. 


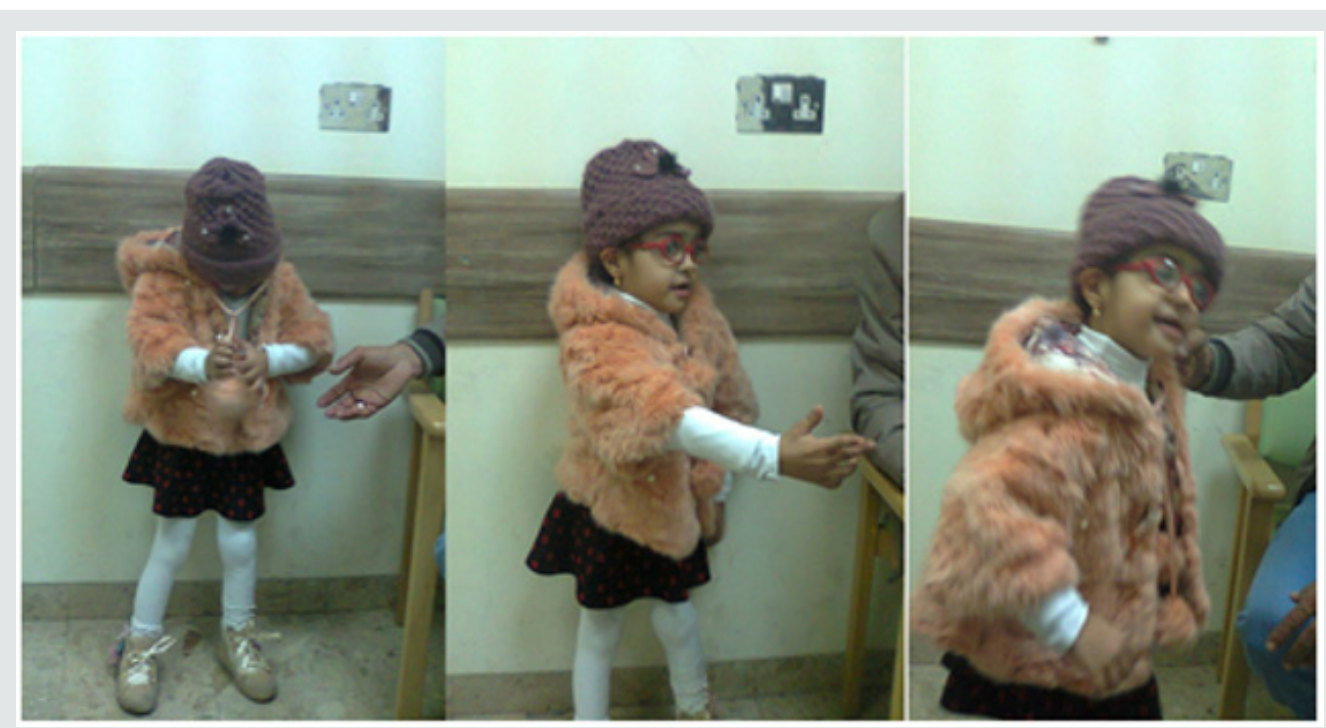

Figure 3: The girl with kernicterus after the second course of treatment.

\section{The Neuroreparative Effects of Cerebrolysin have Been Attributed to [3-5]:}

a) Inhibition of apoptosis.

b) Improving synaptic plasticity and induction of neurogenesis.

c) Augmenting the proliferation, differentiation, and migration of adult.

d) subventricular zone neural progenitor stem cells, contributing to neurogenesis.

e) Induction of stem-cell proliferation in the brain.

Citicoline (cytidine diphosphate choline) is a mononucleotide made of ribose, pyrophosphate, cytosine and choline is a watersoluble naturally occurring substance that is generally grouped with the $\mathrm{B}$ vitamins. It is also considered a form of the essential nutrient choline. It is a safe substance with generally minor side effects which may include digestive intolerance after oral administration [6]. An accumulating research evidence suggests that citicoline is endowed with interesting pharmacological properties that can make it useful in the treatment of various disorders that has no universally accepted effective treatment including neurological conditions such as Parkinson's disease, brain ischemia, hemorrhagic stroke, Alzheimer's disease; and ocular condition such as glaucoma, nonarteritic ischemic neuropathy and amblyopia [6].

\section{The Neuro-Protective Effects of Citicoline Were Attributed to the Followings [6]: \\ a) Preservation of cardiolipin and sphingomyelin \\ b) Preservation of arachidonic acid content of phosphatidylcholine and \\ c) phosphatidylethanolamine.}

d) Partial restoration of phosphatidylcholine levels.

e) Stimulation of glutathione synthesis and glutathione reductase activity.

f) Reduction of phospholipase A2 activity.

g) Increasing glucose metabolism in the brain.

h) Increasing cerebral blood flow.

Reducing oxidative stress and preventing excessive inflammatory response in the brain by inhibiting the release of free fatty acids and reducing blood brain barrier breakdown. Enhances cellular communication by increasing the availability of neurotransmitters, including acetylcholine, norepinephrine, and dopamine. Lowering increased glutamate concentrations and increasing the decreased ATP concentrations induced by ischemia. Citicoline increases dopamine receptor densities, and therefore could improve memory impairment resulting from poor environmental conditions. Citicoline could also improve focus and mental energy and could be useful in the treatment of attention deficit disorder. Citicoline has also been shown to improve visual function in patients with glaucoma [7].

\section{Conclusion}

The novel use of intramuscular cerebrolysin and citicoline in a patient with kernicterus was safe and effective.

\section{References}

1. Brodersen R (1977) Prevention of kernicterus, based on recent progress in bilirubin chemistry. Acta Paediatr Scand 66(5): 625-634.

2. Gourley G (1997) Bilirubin metabolism and kernicterus. Adv Pediatr 44: 173.

3. Al-Mosawi AJ (2018) A novel therapeutic approach for the treatment of brain atrophy. (1 $1^{\text {st }}$ edn); LAP Lambert Academic Publishing, Germany.

4. Al-Mosawi AJ (2018) A novel therapeutic approach for idiopathic mental retardation. ( $1^{\text {st }}$ edn); LAP Lambert Academic Publishing, Germany. 
5. Al-Mosawi AJ (2019) The use of cerebrolysin and citicoline in autism and Asperger syndrome. J Bio Innov 8(1): 99-108.

6. Al-Mosawi AJ (2019) New Therapies for the Treatment of Spastic Cerebral Palsy. Med J Clin Trials Case Stud 3(2): 1-9.
7. Al-Mosawi AJ (2019) Citicoline research progress. (1 $1^{\text {st }}$ edn); LAP Lambert Academic Publishing, Germany.
(C) (P) This work is licensed under Creative

To Submit Your Article Click Here:

Submit Article

DOI: $10.32474 / 0 J N B D .2019 .03 .000151$

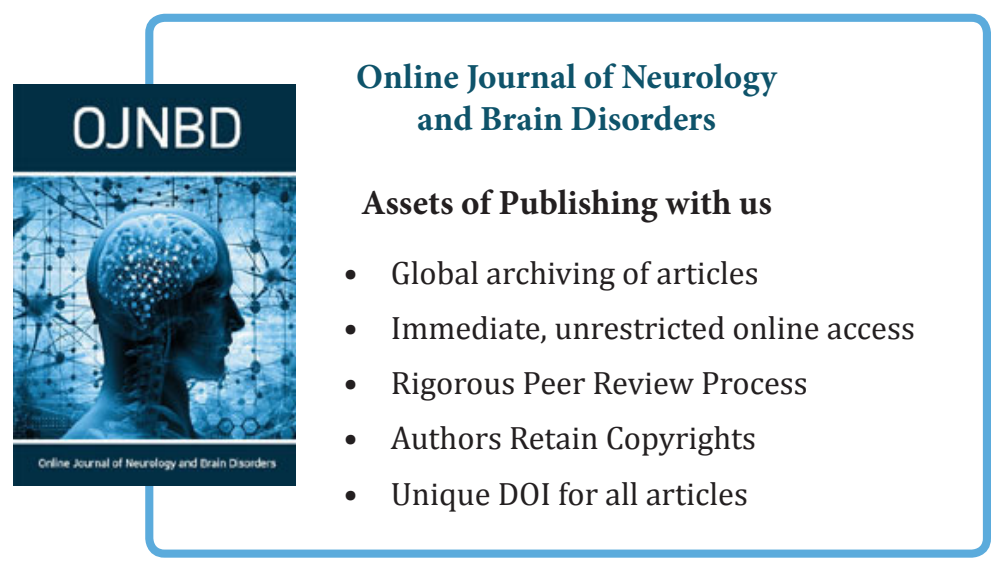

